\title{
Robotic propofol sedation integrating a decision support system specifically designed for patients undergoing a transcatheter aortic valve implantation.
}

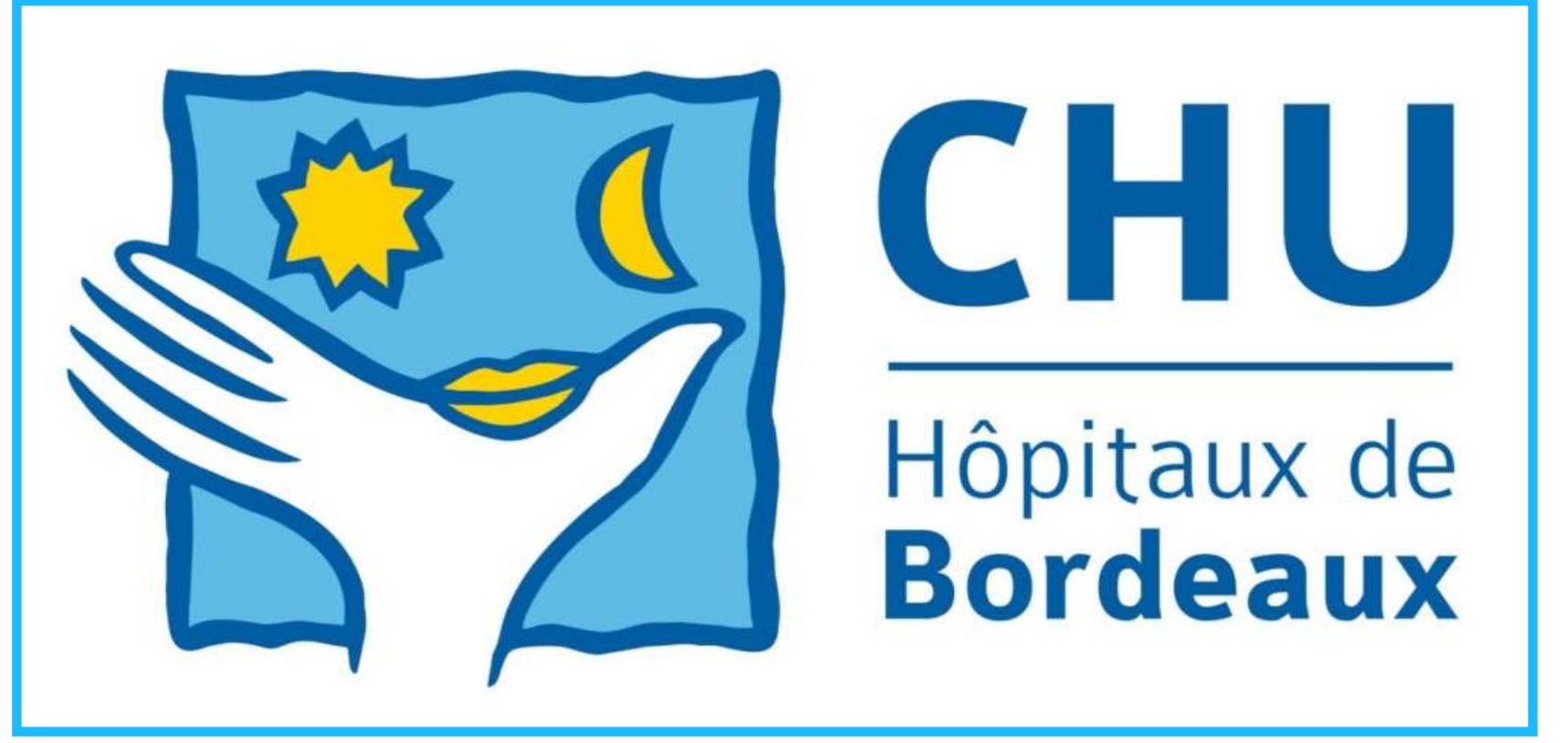

\author{
MION Stefano, Zaouter C. , Leroux L., Remy A., Hemmerling T. , Ouattara A. \\ *Service d'Anesthésie-réanimation II, Haut-Lévêque hospital, CHU Bordeaux, \\ France.Stf.mion@gmail.com
}

\section{Introduction}

On one hand, closed loop systems for propofol sedation have been demonstrated to be safe and reliable for general anesthesia (1), while on the other hand transcatheter aortic valve implantation (TAVI) is . Closed loop systems have never been described in the specific field of TAVI procedures.

\section{Study Objective}

Prospective determination of the feasibility and clinical performance of robotic propofol sedation in patients undergoing TAVI.

\section{Table 1: Clinical performance of hypnosis control}

\section{Clinical performance}

$\%$ of Excellent control

$21,5(12,5)$

$\%$ of Very Good control

$28,6(8,8)$

$\%$ of Good control

$19,2(9,5)$

$\%$ of inadequate control

$30,7(12,7)$

\section{Controller performance}

MDPE (\%)

$-2,4 \pm 11,6$

MDAPE $(\%)$

$23,8 \pm 4,9$

Wobble $(\%)$

$20,3 \pm 4,4$

Divergence (\%.min-1)

$-0,13 \pm 0,15$

Mean (SD) or median (IQR) datas; MDPE = median performance error; MDAPE = median absolute performance error.

\section{Table 2: Critical events per hour of sedation}

Critical respiratory events

$(\mathrm{n}=15)$

Low SpO2 (alarms.h-1)

$0,6(0,6$ to 1,2$)$

Low RR (alarms.h-1)

$2,4(2,1$ to 3,2$)$

\section{Critical hemodynamic events}

Low MAP (alarms.h-1)

$(\mathrm{n}=6)$

Median datas (IQR).

$\mathrm{SpO} 2$ = peripheral oxygen saturation; RR respiratory rate; MAP mean arterial pressure

\section{Methods}

20 consecutive patients scheduled for TAVI were enrolled. Propofol was administered using closed loop feedback control. The control variables were the BIS, hemodynamic and respiratory adverse events which were defined as mean arterial pressure $<60 \mathrm{mmHg}$ and/or heart rate $<40$ bpm and $\mathrm{SpO} 2<92 \%$ and/or respiratory rate $<8 / \mathrm{min}$, respectively.

The clinical performance of hypnosis control was the efficacy to maintain a bispectral index (BIS) of 65 without manual override. The BIS values were stratified into four categories: 'excellent', 'very good', 'good', and 'inadequate' hypnosis control defined as BIS values $<10 \%$, ranging from 11 to $20 \%$, ranging from 21 to $30 \%$ or $>30 \%$ of the target value, respectively. The controller performance was assessed using the varvel's tests (2) The number of critical events per hour of sedation were also recorded.

\section{Results}

According to our above suggestions, robotic sedation was successful in 19 patients $(95 \%$, CI 99\% : $68 \%$ to $100 \%$ ) of the patients undergoing TAVI. One patient was excluded from the final analysis because of a conversion to general anesthesia secondary to a cardiac arrest caused by a pericardial tamponade which required manual override over the system.

The clinical performances of hypnosis described in table 1 allowed an 'excellent' to 'good' control during $71 \%$ (99\% CI: $58 \%$ to $82 \%$ ) of the sedation time. The occurrences of the critical events per hour of sedation are displayed in table 2 .

\section{Conclusions}

The automated closed loop sedation system tested in patients undergoing TAVI was feasible with a satisfactory clinical performance. 